\title{
Untersuchungen über Abietinsäure
}

\author{
(II. Mittheilung) \\ von \\ Dr. Heinrich Mach. $\uparrow$ \\ Aus den chemischen Laboratorien der k. k. deutsehen Universität in Prag und \\ der k. Universität in Göttingen.
}

(Mit 1 'Textfigur.)

(Vorgelegt in der Sitzung am 16. November 1894.)

In der ersten Mittheilung ${ }^{1}$ wurde der Beweis dafür erbracht, dass der krystallisirende Bestandtheil, der nach verschiedenen Verfahrungsweisen aus verschiedenen amerikanischen Colophoniumsorten isolirt werden kann, unter allen Umständen identisch und eine einheitliche Substanz ist, dass ihr aber keine der bisher für dieselbe in Vorschlag gebrachten Formeln zukommt. Die Zusammensetzung der Substanz, für welche der Name Abietinsäure beibehalten worden ist, entspricht vielmehr, nach zahlreichen, von Präparaten, die auf verschiedenen Wegen aus mehreren Colophoniumsorten gewonnen worden waren, ausgeführten Analysen und ebullioskopischen Moleculargewichtsbestimmungen, der Formel $\mathrm{C}_{19} \mathrm{H}_{28} \mathrm{O}_{2}$.

Im Nachstehenden wird über das seither fortgesetzte Studium der Abietinsäure berichtet. ${ }^{2}$

1 Sitzungsberichte der kaiserl. Akademie der Wissenschaften in Wien, mathem.-naturw. Classe, Bd. 102, Abth. II. b, S. 184 und Monatshefte für Chemie, 1893, S. 186.

2 Die vorliegende Arbeit des leider so früh verstorbenen, hochbegabten und vielversprechenden jungen Autors konnte nach dessen ausführlichen nachgelassenen Aufzeichnungen nahezu wortgetreu zusammengestellt werden.

Goldschmiedt. 
Reine Abietinsäure stellt, wie bereits mitgetheilt wurde, farblose, zugespitzte Krystalle vor, die scharf bei $153-154^{\circ}$ schmelzen; sie ist unlöslich in Wasser, hingegen leicht löslich in wässerigen Alkalien, Äthyl-, Methylalkohol, Äther, Aceton, Eisessig, Chloroform, Schwefelkohlenstoff und Benzol; bei allen diesen Lösungsmitteln ist die Löslichkeit in der Wärme bedeutend grösser als in der Kälte. Durch langsames Verdunsten einer absolut alkoholischen Lösung wurden mehrmals grössere messbare Krystalle erhalten.

Herr H. Graber, Assistent am mineralogischen Institute der Universität in Prag, hat die krystallographische Untersuchung dieser Krystalle durchgeführt und theilt darüber Folgendes mit: Die erste krystallographische Untersuchung der Abietinsäure rührt von M. Siewert ${ }^{1}$ her; er beschreibt die Krystalle als lanzettförmige, dünne, zu Krystallbüscheln vereinigte Blättchen, deren Winkel an der Spitze $45^{\circ}$ beträgt. Die Krystalle, die er erhielt, erreichten eine Länge von 6-8 mm bei einer Dicke von $1 \mathrm{~mm}$. Sie waren "weiss ", was wohl "farblos " bedeuten soll. Nach Siewert's früherer Ansicht gehören die Krystalle, die eine dreiseitige Form besitzen, dem ein- und eingliedrigen Systeme an, wwas aber durch die hemiëdrische Ausbildung sehr in Frage gestellt wird «. Die Form der Krystalle entspricht ungefähr der in der Figur, nur mit dem Unterschiede, dass die Endfläche 001 nicht vorhanden ist, die Krystalle also dreiseitige, spitze Blättchen darstellen. Inwieweit die von Siewert gefundenen Winkelwerthe mit den der übrigen Beobachter übereinstimmen, ist aus der Tabelle ersichtlich.

In den Sechziger Jahren untersuchte v. Lang ${ }^{2}$ die Krystalle der von Maly dargestellten Säure. Auch er beschreibt sie als dünne, spitze Blättchen, die an einem Ende stets abgebrochen waren. Das Krystallsystem ermittelt v. Lang triklin.

Im Jahre 1880 unternahm Wulf ${ }^{3}$ eine Untersuchung der von Kelbe aus Harzöl dargestellten Abietinsäure. Auch diese Krystalle sind als trikline bezeichnet worden.

1 Zeitschr. für die gesammten Naturwissenschaften, von Giebel und Hein $z, 14$, S. 311 , Taf. II.

2In Maly's IV. Mittheilung, Joumal für prakt. Chemie, 96, S. 164.

3 In Kelbe's Mittheilung, Ber. der deutschen chem. Gesellsch., 13, S. 888. 
Fock' untersuchte eine nach Flückiger's Methode dargestellte Säure. Das Krystallsystem ist nach ihm monoklin; Hemimorphie nicht unwahrscheinlich. Auch die von Wulf und Fock gemessenen Krystalle waren an einem Ende abgebrochen.

Die, Gegenstand vorliegender Untersuchung bildenden Krystalle sind durch langsames Verdunstenlassen einer äthylalkoholischen Lösung der Säure erhalten worden. Es sind theils ringsum deutlich ausgebildete, theils abgebrochene, farblose, bis $5 \mathrm{~mm}$ grosse und nach 100 tafelförmig ausgebildete Individuen (Figur). Fasst man die kleine Fläche senkrecht zur Symmetrieebene als 001 auf, so ergeben sich für die übrigen Flächen die Symbole $1 \overline{1} 0$ und $1 \overline{1} 0$

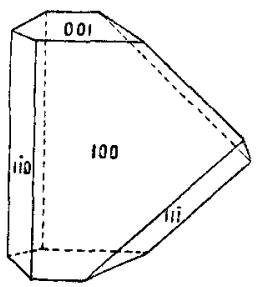
des aufrechten Prismas, $\overline{1} 11$ und $11 \overline{1}$ der positiven Pyramide, ferner 100 und $\overline{1} 00$ als Querflächen.

Die Signale waren meist gut, ausgenommen die der stark parquettirten Querfläche 100 und $\overline{1} 00$.

Krystallsystem: monoklin-hemimorph.

Zur Bestimmung des Axenverhältnisses wurden folgende aus den Mittelwerthen der besten Messungen sich ergebenden Winkel benutzt:

$$
\begin{aligned}
& 100: 1 \overline{1} 0=47^{\circ} 10^{\prime} \\
& 001: 1 \overline{10}=7511 \\
& 001: \overline{1} 10=6018
\end{aligned}
$$

Axenverhältniss:

$$
\begin{gathered}
\beta=112^{\circ} 19^{\prime} \\
a: b: c=1 \cdot 1866: 1: 0 \cdot 9899 ;
\end{gathered}
$$

nach Fock:

$$
\begin{gathered}
\beta=112^{\circ} 28^{\prime} \\
a: b: c=1 \cdot 1811: 1: ?
\end{gathered}
$$

Aus der nachstehenden Tabelle ist ersichtlich, dass die von Mach dargestellte Säure krystallographisch mit der von Fock gemessenen vollkommen identisch ist, höchst wahr-

1 Groth's Zeitschr. für Krystallographie, 1885, VII, S. 58. 


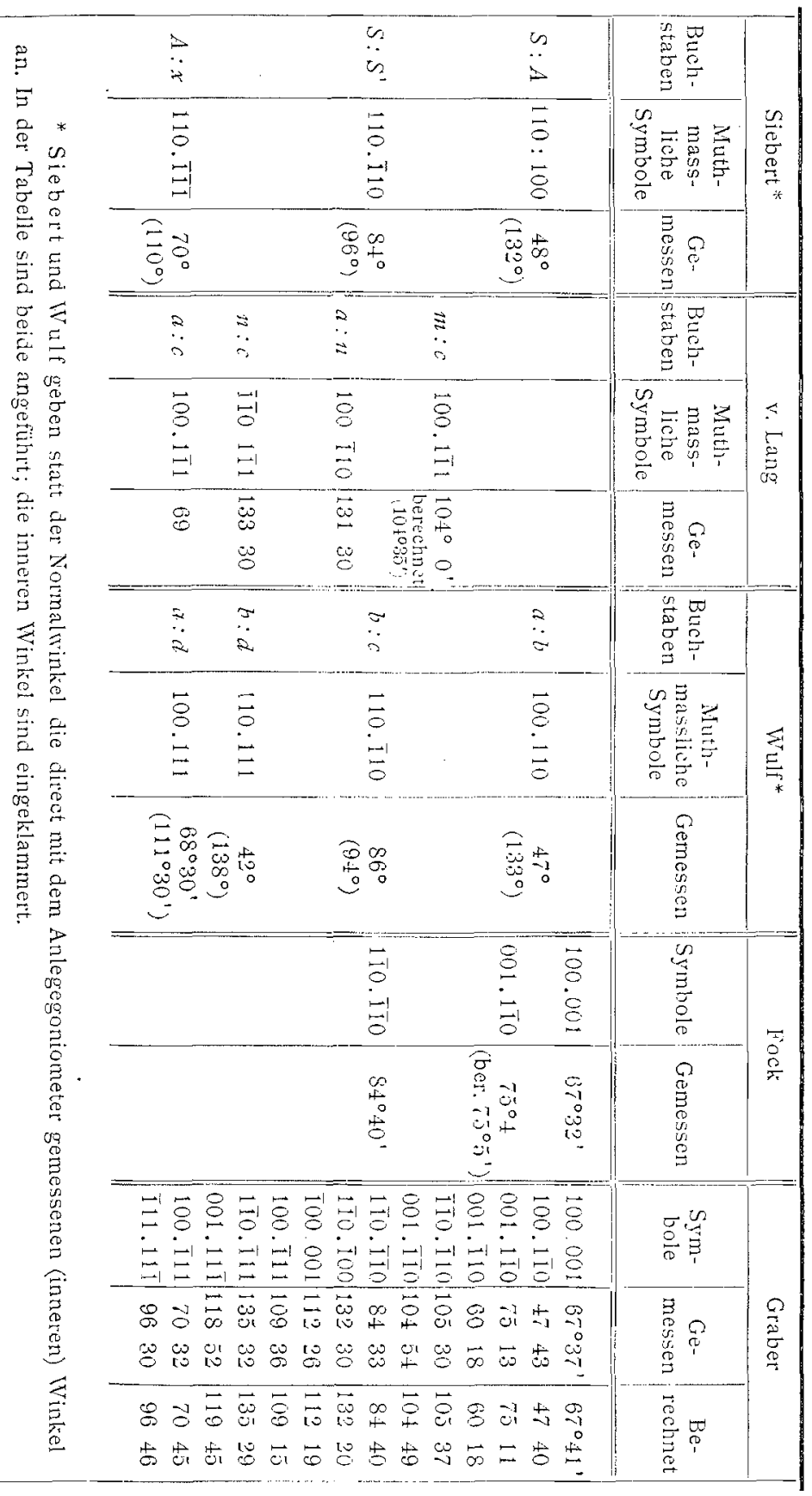


scheinlich stimmt sie auch mit der von $\mathrm{v}$. Lang und von Wulf gemessenen überein. Die Winkelunterschiede bei den von $v$. Lang und Wulf untersuchten Krystallen sind wohl nur auf schlechte Beschaffenheit der Flächen zurückzuführen.

Eine unvollkommene Spaltbarkeit ist nach 001 und 100 beobachtet worden. Nach 001 waren an einigen Krystallen Spaltrisse entstanden. Wulf hat Spaitbarkeit nach der tafelförmigen Fläche beobachtet; v. Lang und Fock geben keine Spaltbarkeit an.

Die Ebene der optischen Axe liegt parallel zur Längsfläche 010 .

Auf der Querfläche 100 wurde, am Rande des Gesichtsfeldes, das Austreten einer optischen Axe beobachtet; die nämliche Beobachtung machte auch v. Lang.

Das Bild der andern optischen Axe wird sichtbar auf einer parallel 001 geschliffenen Platte. Dieses scheint geneigt gegen die Normale zu dieser Fläche. Fock bemerkte durch seine Fläche 001 ebenfalls das Austreten einer optischen Axe unter ähnlichen Umständen.

An der zu 001 parallelen Platte ist überdies der negative Charakter der Doppelbrechung bestimmt worden.

Dispersion $p<v$ um die negative Mittellinie a. Die Axe grösster Elasticität a bildet auf Platten parallel 010 mit der Kante 010.100 einen Winkel von circa $13^{\circ} \mathrm{im}$ Sinne des Uhrzeigers $\ll$.

Die Abietinsäure zeigt einige sehr charakteristische Farbenreactionen, welche sie mit dem Gallen-Cholestearin gemeinsam hat. Wird eine geringe Menge Abietinsäure in trockenem Chloroform gelöst, etwas Essigsäureanhydrid, hierauf einige Tropfen concentritte Schwefelsäure zugesetzt, so tritt zunächst eine purpurrothe, rasch durch violett und blau in tiefes Schwarzroth übergehende Fürbung auf, welche schliesslich einer grünlichen Missfärbung Platz macht. Ganz ähnliche Reactionen geben noch viele andere Körper, wie z. B. Amyrin, Gentiol, Urson, Vitin und viele Glieder der Terpengruppe. W. Seifert ${ }^{1}$ hat kürzlich Beiträge zu dieser Reaction geliefert, indem

1 Monatshefte für Chemie, 1893, S. 728. 
er die Absorptionsspectra der Lösungen von Vitin, AbietinSäure und Urson in Schwefelsäure und in Schwefelsäure und Essigsäureanhydrid untersuchte; in seiner Abhandlung finden sich dieselben graphisch dargestellt.

Wird Abietinsäure auf einem Uhrglase mit 3 Volumen concentrirter Salzsüure und 1 Volumen Eisenchloridlösung vorsichtig zur Trockene gebracht, so löst sich ein Theil der Substanz auf, während die ungelöst gebliebenen Kryställchen eine violette, dann ins Bläuliche sich ziehende Färbung annehmen. Auch diese Reaction theilt die Abietinsäure mit dem Cholestearin, dem Gentiol und dem Urson, wie W. Gintl ${ }^{1}$ jun. nachgewiesen hat.

Beim Verdampfen von Abietinsäure mit Salpetersäure zur Trockene und Befeuchten des hiebei bleibenden Rückstandes mit Ammoniak tritt Rothfärbung auf.

Abietinsäure, auch das reinste Präparat, löst sich in kalter, concentrirter Schwefelsäure mit rother Farbe auf; wird eine solche Lösung mit Chloroform kräftig durchschüttelt, so färbt sich die Chloroformschichte rosa, während die Schwefelsäure nach einiger Zeit eine schwache grüne Fluorescenz. annimmt.

Abietinsäure ist, wie schon Haller beobachtet hat, optisch activ, und zwar stark linksdrehend.

Einige vorläufige Bestimmungen ergaben:

1. Abietinsäure aus Colophonium K. ${ }^{2}$ mit Salzsäure dargestellt) in alkoholischer Lösung. (Schmelzpunkt der Säure $\left.153-154^{\circ}\right)$

$$
\begin{aligned}
c & =6 \cdot 4816 \\
\alpha & =-3 \cdot 693^{\circ} \\
l & =1 \mathrm{dm} \\
{[\alpha]_{D} } & =-56 \cdot 97
\end{aligned}
$$

nach weiterem einmaligen Umkrystallisiren in alkoholischet Lösung (Schmelzpunkt unverändert):

1 Monatshefte für Chemic, 1893, S. 266.

2 Siehe I. Mittheilung. 
I.

$$
\begin{aligned}
c & =6 \cdot 3475 \\
\alpha & =-4 \cdot 2495^{\circ} \\
l & =1 \mathrm{dm} \\
{[x]_{D} } & =-66 \cdot 94
\end{aligned}
$$

II. $-4 \cdot 2745^{\circ}$

$1 \mathrm{dm}$

$-67 \cdot 34$

nach weiterem dreimaligem Umkrystallisiren (Schmelzpunkt unverändert):

$$
\begin{aligned}
c & =3 \cdot 2448 \\
\alpha & =-2 \cdot 163^{\circ} \\
l & =1 d m \\
{[\alpha]_{D} } & =-66 \cdot 66
\end{aligned}
$$

Haller fand für seine reinste Abietinsäure $[a]_{D}=-53$, Dietrich \pm 48 .

\section{Salze der Abietinsäure.}

Schon in der ersten Abhandlung wurde erwähnt, dass vielfache, unter den verschiedensten Bedingungen ausgeführte Versuche, neutrale Salze der Abietinsäure zu erhalten, vergeblich gewesen sind; es wurden stets Substanzen, von wechselnder, mit der Formel der Abietins äure nicht im Einklange stehender Zusammensetzung erzielt; nur ein gut krystallisirendes saures Kaliumsalz $\mathrm{C}_{19} \mathrm{H}_{27} \mathrm{O}_{2} \mathrm{~K}, 3 \mathrm{C}_{19} \mathrm{H}_{28} \mathrm{O}_{2}$, das auf zwei verschiedenen Wegen dargestellt werden konnte, ist schon damals beschrieben worden. Es ist im Verlaufe der fortgesetzten Versuche schliesslich gelungen, auch das neutrale Kalium- und $B$ arium-, sowie ein saures Ammonsalz zu gewinnen.

Neutrales Kaliumsalz. Es wird erhalten, wenn eine alkoholisch-ätherische Lösung der A bietinsäure durch einige Tage mit überschüssigem Kaliumcarbonat gekocht wird: $18 g$ Abietinsäure, $4 \cdot 4 g$ reines, trockenes, natriumfreies Kaliumcarbonat, $120 \mathrm{~cm}^{3}$ absoluter Alkohol und $100 \mathrm{~cm}^{3}$ Äther wurden 170 Stunden am Rückflusskühler gekocht. Nach dieser Zeit ist das Kaliumcarbonat bis auf einen geringen zurückbleibenden Überschuss gelöst. Die filtrirte Lösung scheidet im Vacuum blumenkohlartige Krystallaggregate oder lange, seideglänzende, zu breiten Büscheln vereinigte Krystalle aus. Diese sind rein weiss, in kaltem, leichter in heissem Wasser, in Alkohol und in 
H. Mach,

Äther löslich. Das Salz lässt sich wohl aus Alkohol, nicht aber aus Wasser umkrystallisiren. Eine wässerige Lösung scheidet sowohl bei Luftzutritt, als auch im Vacuum eine seifenartige, fast ölige, weisse Masse aus. Wie aus den nachstehenden Analysen hervorgeht, ist das $\mathrm{Salz}$ nicht rein, und die erhaltenen Werthe dürfen nicht als Stütze für die Formel $\mathrm{C}_{19} \mathrm{H}_{26} \mathrm{O}_{2}$ herangezogen werden.

Es ist wohl sehr wahrscheinlich, dass das Salz während des langen Kochens Sauerstoff aufgenommen hat. Trotz wiederholter Reinigung durch Umkrystallisiren konnte es nicht in bessere Form gebracht werden. Immerhin zeigen die Analysen, dass wirklich ein neutrales Salz $\mathrm{C}_{19} \mathrm{H}_{27} \mathrm{O}_{2} \mathrm{~K}$ vorliegt:

I. $0.2285 \mathrm{~g}$ Substanz, im Vacuum über Schwefelsäure getrocknet, gaben $0.5782 \mathrm{~g}$ Kohlensäure, $0.1741 \mathrm{~g}$ Wasser und $0.0333 \mathrm{~g}$ Kaliumoxyd.

II. $0 \cdot 2152 g$ Substanz, wie bei I getrocknet, gaben $0.5412 g$ Kohlensäure, $0.1624 \mathrm{~g}$ Wasser und $0.0318 \mathrm{~g}$ Kaliumoxyd.

III. $0.2438 \mathrm{~g}$ Substanz, im Vacuum bei $120^{\circ}$ getrocknet, gaben $0.6111 \mathrm{~g}$ Kohlensäure, $0.1847 \mathrm{~g}$ Wasser und $0.0368 \mathrm{~g}$ Kaliumoxyd.

In 100 Theilen:

\begin{tabular}{|c|c|c|c|}
\hline & Gefunder & & Jerechnet \\
\hline I. & II. & III. & tür $\mathrm{C}_{19} \mathrm{H}_{17} \mathrm{O}_{32} \mathrm{~h}$ \\
\hline C ...09:04 & $68 \cdot 60$ & $68 \cdot 68$ & $69 \cdot 94$ \\
\hline$H \ldots . . .8 \cdot 46$ & $8 \cdot 38$ & $8 \cdot 43$ & $8 \cdot 28$ \\
\hline K....12.09 & $12 \cdot 95$ & $12 \cdot 30$ & $11 \cdot 96$ \\
\hline
\end{tabular}

Es ist versucht worden, nach zahlreichen anderen Methoden neutrales Kaliumsalz darzustellen; sümmtliche in dieser Richtung angestellten Versuche ergaben jedoch ein negatives Resultat.

Neutrales Bariumsalz. Aus dem eben beschriebenen Kaliu m salze kann eine Ba riu merbindung dargestellt werden, welche, wie die weiter unten angeführte Analyse lehrt, die Zusammensetzung eines neutralen Salzes hat. Eine Lösung von abietinsaurem Kalium in Wasser wurde mit einer ebensolchen von reinstem Bariumacetat versetzt, die hiebei 
entstehende weisse voluminöse Fällung abgesaugt und sehr sorgfältig mit Wasser gewaschen. Das Salz stellt ein amorphes weisses Pulver dar, welches sich bei Luftzutritt rasch gelb fürbt.

I. $0.2050 \mathrm{~g}$ Substanz im Vacuum über Schwefelsäure getrocknet gaben $0 \cdot 4830 \mathrm{~g}$ Kohlensäure, $0 \cdot 1480 \mathrm{~g}$ Wasser und $0 \cdot 0440 \mathrm{~g}$ Bariumoxyd.

II. $0 \cdot 7901 \mathrm{~g}$ Substanz gaben mit Schwefelsäure abgeraucht $0 \cdot 2556 \mathrm{~g}$ Bariumsulfat.

In 100 Theilen:

\begin{tabular}{|c|c|c|}
\hline Gef & den & $\begin{array}{l}\text { Berechnet fü̈ } \\
\left(\mathrm{C}_{19} \mathrm{H}_{27} \mathrm{O}_{3}\right)_{2} \mathrm{~B}\end{array}$ \\
\hline C.....64.25 & - & $64 \cdot 14$ \\
\hline$\ldots \ldots 7 \cdot 63$ & — & $7 \cdot 59$ \\
\hline $\mathrm{Ba} \ldots \ldots \ldots 19 \cdot 22$ & $19 \cdot 02$ & $19 \cdot 27$ \\
\hline
\end{tabular}

Saures Ammoniumsalz. Abietinsäure löst sich leicht in warmen wässerigem oder alkoholischem Ammoniak; die Lösungen erstarren beim Erkalten zu einer steifen Gallerte, welche nicht in krystallisirte Form zu bringen ist. Es gelingt aber leicht, nach den beiden im Folgenden beschriebenen Methoden ein schön krystallisirtes Ammoniumsalz von der Zusammensetzung $\mathrm{C}_{19} \mathrm{H}_{27} \mathrm{O}_{2}\left(\mathrm{NH}_{4}\right) \cdot \mathrm{C}_{19} \mathrm{H}_{28} \mathrm{O}_{2}$ darzustellen.

$1.5 \mathrm{~g}$ Abietins äure werden mit $10 \mathrm{~g}$ käuflichem Ammonitumcarbonat und $35 \mathrm{~cm}^{3}$ Alkohol 4 Stunden unter Ersatz des verdampfenden Alkohols bei circa $60^{\circ}$ digerirt. Die Lösung färbt sich gelb und scheidet beim Erkalten grosse, zu Drusen vereinigte prismatische Krystalle $a b$, die nach einmaligem Umkrystallisiren rein weiss und analysenrein sind.

2. Dasselbe Salz wird erhalten, wenn durch eine kalte alkoholische Lösung von Abietinsäure $(1: 6)$ ein ziemlich rascher Strom von Kohlensäure und Ammoniak geleitet wird; die Lösung scheidet, nach dem Verjagen des überschüssigen Ammoniaks auf dem Wasserbade, im Vacuum eine reichliche Krystallisation des oben beschriebenen Salzes ab.

Das Salz ist sehr beständig, leicht löslich in warmem Alkohol oder Äther, unlöslich in Wasser. 
I. $0 \cdot 2347 \mathrm{~g}$ Substanz (nach Methode 1 dargestellt), im Vacuum getrocknet, gaben $0.6608 \mathrm{~g}$ Kohlensäure und $0 \cdot 2086 g$ Wasser.

II. $0.2734 \mathrm{~g}$ Substanz (nach Methode 2 dargestellt), im Vacuum getrocknet, gaben $0 \cdot 7705 \mathrm{~g}$ Kohlensäure und $0.2430 \mathrm{~g}$ Wasser.

In 100 Theilen:

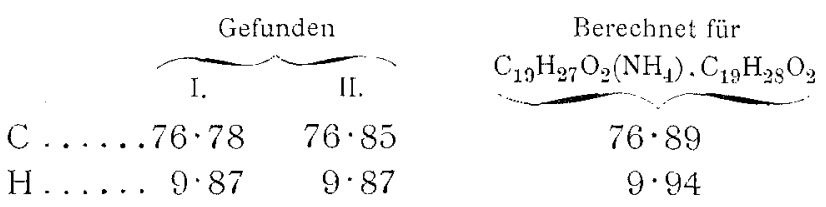

Nach verschiedenen Methoden und unter Einhaltung verschiedener Versuchsbedingungen sind eine Anzahl von Calcium-, Barium-, Kupfer- und Silberverbindungen erhalten worden, deren Elementaranalyse deutlich erkennen liess, dass dieselben vollkommen inhomogene Körper darstellten. Man kann sich die Bildung solcher Verbindungen einmal durch den sehr schwach sauren Charakter der Abietinsäure und dann durch den dissociirenden Einfluss, welchen die in Anwendung gekommenen Lösungsmittel - Alkohol und Wasser - auf die entstandenen Verbindungen auszuüben vermögen, erklären. Die schwache Acidität der Säure legte den Gedanken nahe, dass dieselbe mit der stärksten verfügbaren Base - Tetramethylammoniumhydroxyd -- eine neutrale Verbindung zu bilden im Stande sein durite.

$\mathrm{Es}$ ist in der That gelungen, eine in prachtvollen, oft mehrere Centimeter langen und bis $8 \mathrm{~mm}$ breiten glashellen Prismen krystallisirende, wasserlösliche Tetramethy lammoniumverbindung darzustellen; sobald aber die Krystalle aus der absolut alkoholischen Mutterlauge entfernt werden, trüben sie sich nach wenigen Minuten und zerfallen zu einem gelben, stark nach Trimethylamin riechenden Pulver, welches bei der Analyse die nachstehenden Werthe ergab:

$0.2125 \mathrm{~g}$ Substanz gaben $0.5537 \mathrm{~g}$ Kohlensäure und $0.2010 \mathrm{~g}$ Wasser. 
In 100 Theilen:

$\begin{array}{cc}\text { C. } \ldots \ldots 71 \cdot 0 \dot{6} & \begin{array}{c}\text { Berechnet für } \\ \mathrm{C}_{19} \mathrm{H}_{27} \mathrm{O}_{2}\left(\mathrm{~N}\left(\mathrm{CH}_{3}\right)_{4}\right)\end{array} \\ \mathrm{H} \ldots \ldots 10.51 & 76 \cdot 45 \\ & 10 \cdot 80\end{array}$

Abietinsäurechlorid. $11 \mathrm{~g}$ Abietinsäure wurden in trockenem Chloroform gelöst, und diese Lösung ist bei Ausschluss feuchter Luft zu $10 \mathrm{~g}$ Phosphorpentachlorid hinzugefügt worden; es tritt eine mässige Reaction unter schwacher Erwärmung ein. Das Lösungsmittel und das gebildete Phosphoroxychlorid werden im Vacuum abdestillirt. Der Rückstand stellt ein gelbes, zähes, dickflüssiges Ö1 dar, welches nicht umlirystallisirbar ist und bei -10 bis $12^{\circ}$ fest wird. Es ist im Vacuum nicht unzersetzt destillirbar, sondern liefert hiebei ein dünnes, schwach fluorescirendes $\ddot{O} 1$ von intensivem Isonitrilgeruch; dasselbe erstart bei $-12^{\circ}$ noch nicht und ist vielleicht identisch mit Maly's Abietenen. ${ }^{1}$

Abietinsäureamid. Durch Behandlung des Chlorides in absolut alkoholischer Lösung mit Ammoniak ist ein bei $63^{\circ}$ schmelzendes, nur sehr schwer krystallisirendes A mid erhalten worden; dasselbe in einer zur Analyse brauchbaren Form darzustellen ist nicht gelungen.

Ebensowenig ist es gelungen, das aus dem Säurechlorid mit Anilin dargestellte Anilid und den aus dem Chloride mit Methylalkohol entstehenden Methylester in analysenreiner Form zu gewinnen.

\section{Oxydation von Abietinsäure mit Kaliumpermanganat in alkalischer Lösung.}

Diese Reaction ist bereits von Emmerling ${ }^{2}$ studirt worden; doch hat dieser Forscher dabei nur weitgehende Oxydationsproducte, wie Kohlen-, Ameisen- und Essigsäure beobachtet. Bei mässiger Oxydation wird aber, wie im Fol-

1 Abietene nannte Maly die bei der Destillation von Abietinsäure mit Phosphorpentachlorid entstehenden Kohlenwasserstoffe.

2 Ber. der deutschen chem. Gesellsch., XII, S. 1411. 
genden gezeigt werden soll, glatt und in guter Ausbeute eine Ketonsäure von der Formel $\mathrm{C}_{10} \mathrm{H}_{10} \mathrm{O}_{3}$, gebildet.

$20 \mathrm{~g}$ Abietinsäure wurden in einem Liter zweiprocentiger Kalilauge gelöst und zu dieser Lösung, in kleinen Portionen, eine Auflösung von $20 \mathrm{~g}$ Kaliumpermanganat in $1 \mathrm{l}$ Wasser hinzugefügt. Die Oxydation vollzog sich ziemlich rasch unter Erwärmung. Die vom ausgeschiedenen Braunstein abfiltrirte hellgelbe Lösung wird mit Kohlensäure gesättigt und zur Trockene verdampft. Der Rückstand wird mehrmals mit heissem, absolutem Alkohol extrahirt, wobei ein weisses, hauptsächlich aus Kaliumcarbonat und -oxalat bestehendes Salzgemisch ungelöst bleibt. Der Verdampfungsüukstand des alkoholischen Auszuges wurde in Wasser gelöst; aus dieser Lösung fällt, auf Zusatz von verdünnter Schwefelsäure, ein weisser flockiger Niederschlag, der abgesaugt und mit viel Wasser gewaschen wurde. Das Filtrat enthält keine organische Substanz mehr; es ist demnach der vorliegende Körper das einzige primäre Reactionsproduct. Dic Substanz ist amorph und lässt sich auf keine Weise krystallisirt erhalten, hat sauren Charakter, ist in Wasser unlöslich, leicht löslich in wïsserigen Alkalien, in Ammoniak, Alkohol, Äther und Benzol; eine heiss bereitete Benzollösung erstart beim Erkalten zu einer steifen Gallerte; dieses Verhalien zeigen selbst sebr verdünnte Lösungen. Durch Auflösen in Benzol und Fällen mit Petroläther und Wiederholen dieser Operation kann der Körper gereinigt werden; er stellt dann ein rein weisses, kreidiges Pulver vom Schmelzpunkte $123^{\circ}$ dar und wurde, im Vacuum getrocknet, analysirt:

$0.2317 \mathrm{~g}$ Substanz gaben $0.5558 \mathrm{~g}$ Kohlensäure und $0.1858 \mathrm{~g}$ Wasser.

In 100 Theilen:

$\begin{array}{cc}\text { Geftunden } & \begin{array}{r}\text { Bcrechnet } \\ \text { für } \mathrm{C}_{114} \mathrm{H}_{16} \mathrm{O}_{33}\end{array} \\ \mathrm{H} \ldots \ldots .65 \cdot 42 & 65 \cdot 22 \\ 8.91 & 8.69\end{array}$

Dem Körper scheint demnach die Formel $\mathrm{C}_{10} \mathrm{H}_{16} \mathrm{O}_{3}$ zuzukommen, welche durch Derivate zu stützen bisher nicht 
gelungen ist, da solche in brauchbarer Form nicht erhalten werden konnten. Diese Verbindung dürfte wohl als Ketonsäure anzusprechen sein, denn sie gab in alkalischer Lösung mit Brom behandelt, reichliche Mengen von Tetrabromkohlenstoff, ${ }^{1}$ welcher aus der Reactionsflüssigkeit mit Wasserdampf abgeblasen und dann an seinem Schmelzpunkt 90-91 (Lit. 92:5) erkannt werden konnte; aus der rückständigen alkalischen Lösung kann sonst kein weiteres Zersetzungsproduct isolirt werden; ein grosser Theil der Säure wird unverändert wiedergewonnen.

\section{Verhalten der Abietinsäure in alkalischer Lösung gegen}

\section{Brom.}

$5 \mathrm{~g}$ Abietinsäure werden in circa $200 \mathrm{~cm}^{3}$ schwacher Natronlauge gelöst und dann wird noch so viel Natronlauge zugesetzt, als die Lösung, ohne sich zu trüben, verträgt, hierauf wird so lange Brom in kleinen Portionen eingetragen, bis keine Entfärbung eintritt; während der Operation scheidet sich eine unbedeutende Menge weisser Flocken aus. Zur weiteren Verarbeitung wird die Flüssigkeit mit schwefliger Säure behandelt und schliesslich mit verdünnter Schwefelsäure schwach angesäuert. Hiebei fällt ein weisser, flockiger Niederschlag aus, der abgesaugt, mit viel Wasser gewaschen und dann getrocknet, ein weisses, kreidiges, bromhaitiges Pulver darstellt. Der Körper ist leicht löslich in Alkohol, Äther, Benzol und Chloroform und kann durch mehrmaliges Auflösen in Benzol und Ausfällen mit Petroleumäther bromfrei erhalten werden; er färbt sich bei $130^{\circ}$ braun und zersetzt sich bei $137^{\circ}$ unter stürmischer Gasentwicklung.

$0.1848 \mathrm{~g}$ Substanz, im Vacuum getrocknet, gaben $0.3160 \mathrm{~g}$ Kohlensäure und $0 \cdot 0902 \mathrm{~g}$ Wasser.

In 100 Theilen:

$$
\begin{array}{ccc}
\mathrm{C} \ldots \ldots & 61 \cdot 39 \\
\mathrm{H} \ldots \ldots & 5 \cdot 42 & \begin{array}{r}
\text { Gefunden } \\
\text { Bür } \mathrm{C}_{10} \mathrm{H}_{10} \mathrm{O}_{4}
\end{array} \\
51 \cdot 85
\end{array}
$$

1 Siehe Wallach, Liebig's Annalen, 275, 145. 
Auch bei dieser Verbindung ist es nicht gelungen, brauchbare Derivate darzustellen.

Die Abietinsäure ist in der Literatur häufig mit der Pimarsäure (aus Galipot und Colophonium di Bordeaux) identificirt worden. So sagt z. B. Maly in seiner III. Abhandlung: »Die Pimarsäure scheint nach den vorhandenen Mittheilungen nichts Anderes als Abietinsäure zu sein", und diese nur vermuthungsweise ausgesprochene Ansicht ist in einige Lehrbücher ( $z$. B. Gmelin-Ǩraut) übergegangen, obwohl sie durch die Untersuchungen von Duvernoy und Strecker eine Widerlegung erfahren hat.

Die beiden Säuren sind aber, wie aus den schönen Untersuchungen Vesterberg's einerseits und aus dem Inhalte der I. Mittheilung des Verfassers über Abietinsäure anderseits hervorgeht, zwei vollständig verschiedene Kïrper, wenngleich sie zu einander in naher Beziehung zu stehen scheinen.

Heute kann es nicht mebr zweifelhaft sein, dass die $A$ bietin- und Pimarsäure nicht nur nicht identische, sondern auch nicht isomere Verbindungen sind.

Es war ursprünglich beabsichtigt, eine vollständige vergleichende Untersuchung der beiden in Rede stehenden Säuren vorzunehmen und vor Allem ihr Verhalten bei gleichen Reactionen zu studiren. Leider ist es aber nicht gelungen, aus den im Handel vorkommenden und dem Verfasser zugänglich gewesenen, als "Gallipot bezeichneten Harzsorten Pimarsäure zu isoliren. Die Harze enthielten entweder überhaupt keine krystallisirbaren Bestandtheile, oder es konnte aus ihnen nur Abietinsäure abgeschieden werden. So ist beispielsweise auch ein französisches Colophonium aus Galipot untersucht worden; das in seinem üusseren Ansehen dem amerikanischen Colophonium völlig gleichende Harz war in der Fabrik der Firma Schimmel \& Comp. aus Galipot bereitet worden und lieferte beim Behandeln mit Salzsäure in alkoholischer Lösung Abietins äure in vortrefflicher Ausbeute $(98 \%$ rohe, $34 \%$ analysenreine Substanz). Der Schmelzpunkt lag, wie bei allen früher untersuchten reinen AbietinsäurePräparaten bei $153-154^{\circ}$. 
$0.2866 \mathrm{~g}$ der dreizehnmal umkrystallisirten Substanz gaben $0 \cdot 8301 \mathrm{~g}$ Kohlensäure und $0 \cdot 2535 \mathrm{~g}$ Wasser.

In 100 Theilen:

$$
\begin{aligned}
& \text { Berechnet } \\
& \text { C . . Gefunden } \frac{\text { für } \mathrm{C}_{19} \mathrm{H}_{28} \mathrm{O}}{79 \cdot 99} \quad \frac{16}{99} \\
& \text { H ... } 9 \cdot 83 \quad 9 \cdot 73
\end{aligned}
$$

Auch bei Anwendung von verdünntem Alkohol und Schwefelsäure konnte aus diesem Harze Abietinsäure vom Schmelzpunkte $153-154^{\circ}$ dargestellt werden.

Die Bestimmung des optischen Drehungsvermögens dieser vierzehnmal umkrystallisirten Säure in alkoholischer Lösung ergab Folgendes:

$$
\begin{aligned}
c & =2 \cdot 261 \\
\alpha & =-1 \cdot 560^{\circ} \\
l & =1 \mathrm{dm} \\
{[a]_{D} } & =-69 \cdot 96
\end{aligned}
$$

Her Dr. Vesterberg in Upsala hat dem Verfasser in dankenswerther Weise eine kleine Probe Dextropimarsäure zur Verfügung gestellt. Ihr Schmelzpunkt lag entsprechend Vesterberg's Angaben bei $212^{\circ}$, und sie hat bei der Analyse Zahlen ergeben, die mit den von Vesterberg gefundenen und von der Formel $\mathrm{C}_{20} \mathrm{H}_{30} \mathrm{O}_{2}$ geforderten in vorzüglicher Übereinstimmung stehen:

$0.2072 \mathrm{~g}$ Substanz, im Vacuum getrocknet, gaben $0.6027 \mathrm{~g}$ Kohlensäure und $0 \cdot 1868 g$ Wasser.

In 100 Theilen:

$$
\begin{array}{cc}
\text { Gefunden } & \begin{array}{c}
\text { Berechnet } \\
\text { für } \mathrm{C}_{20} \mathrm{H}_{30} \mathrm{O}_{2}
\end{array} \\
\mathrm{H} \ldots \ldots .79 \cdot 33 & 79 \cdot 47 \\
7 . \ldots 10 \cdot 00 & 9 \cdot 94
\end{array}
$$

Das Ergebniss der vergleichenden Untersuchung der Abietin- und Pimarsäure ist demnach, dass die beiden Säuren nicht identisch und auch nicht isomer sind. Um die festgestellten Verschiedenheiten in ihren Eigenschaften besser hervortreten zu lassen, sollen diese in tabellarischer Form neben einander gestellt werden. 


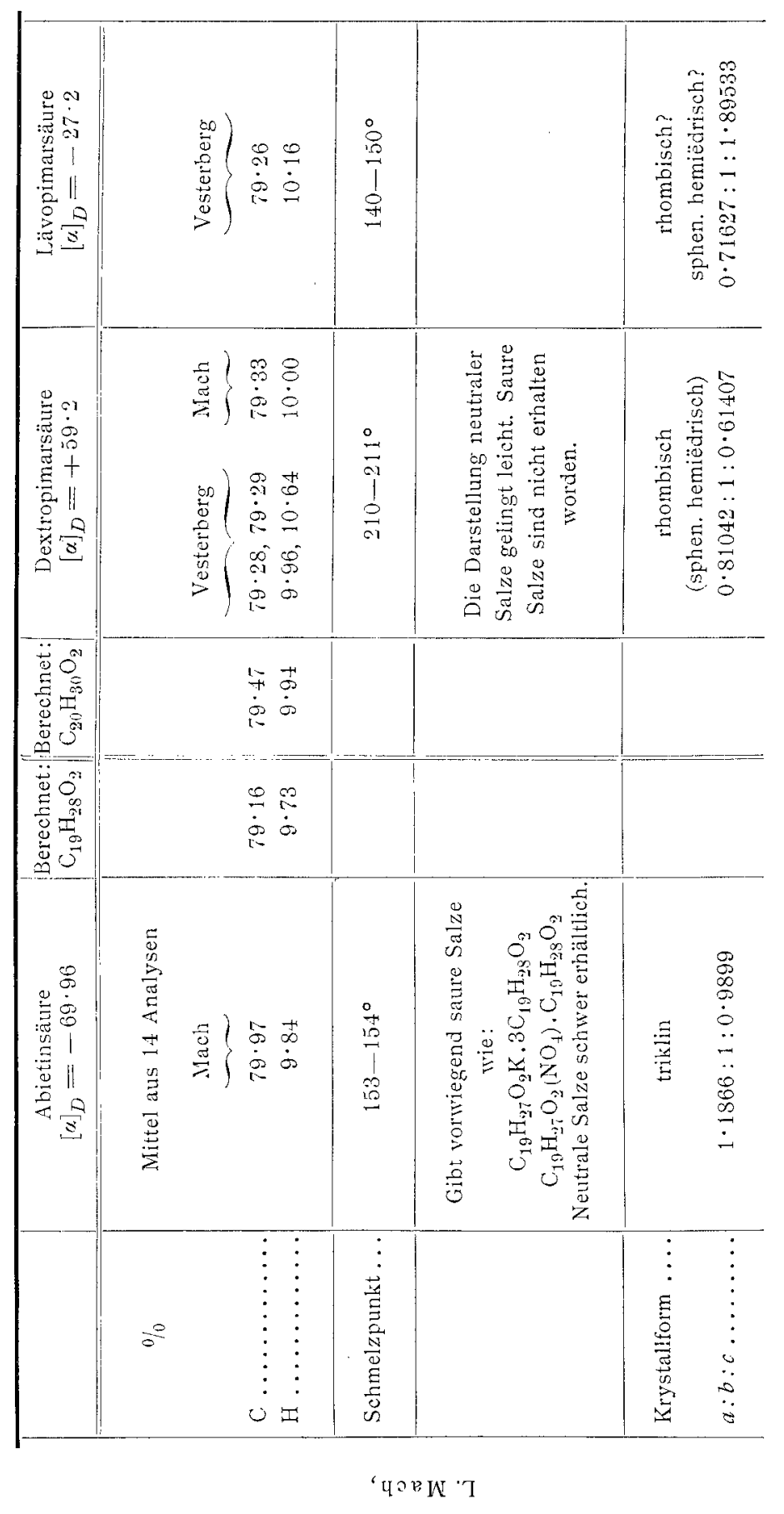




\section{Anhang.}

H. Mach hat sich während seines Aufenthaltes in Göttingen eingehend auch mit dem Studium der Metacopaïvasäure befasst. Anlass hiezu war der Umstand, dass für diese Substanz die Formel $\mathrm{C}_{20} \mathrm{H}_{20} \mathrm{O}_{2}$ aufgestellt worden war, wodurch sie als Isomeres der Pimarsäure erschien. Über diese Untersuchung liegen ausführliche Aufzeichnungen und ein umfangreiches analytisches Materiale vor, von deren eingehenden Mittheilung Abstand genommen wird, weil die noch nicht abgeschlossene Arbeit von anderer Seite fortgesetzt und erst dann in extenso publicirt werden soll. Nachstehend nur ganz kurz die Hauptresultate Mach's.

Reine Metacopaïvasäure wies bei den Analysen einen um mehrere Procente höheren Kohlenstoffgehalt auf, als in der Literatur angeführt wird, sie besitzt die Formel eines Sesquiterpenalkohols $\mathrm{C}_{15} \mathrm{H}_{23} \mathrm{OH}$ und dürfte das Anfangsglied der Cholestearinreihe sein; die in der Literatur als Cholestearine angeführten pflanzlichen und thierischen Cholestearine können als Homologe dieses Körpers aufgefasst werden; das Gallencholestearin dürfte das höchstmoleculare bekannte Glied dieser Reihe sein. Mach nennt die Substanz, von welcher schon $\mathrm{Brix}^{1}$ nachgewiesen hatte, dass sie ein Alkohol ist - er hatte ihr aber die Formei $\mathrm{C}_{20} \mathrm{H}_{28}(\mathrm{OH})_{2}$ zugeschrieben - wegen ihrer unverkennbaren Ähnlichkeit mit Liebermann's ${ }^{2}$ Cholestol - Metacholestol.

Metacholestol krystallisirt wie Gallen-Cholestearin aus wasserhaltigem Alkohol in Blättchen, aus Benzol und Petroleumäther in Nadeln. Es wurde ein Acetat, Propionat und Benzoat dargestellt, welche in ihrer Zusammensetzung mit obiger Formel im Einklange sind. Die Hydroxy lgruppe ist gegen Brom austauschbar, die Verbindung $\mathrm{C}_{15} \mathrm{H}_{23} \mathrm{Br}$ ist, wenn auch schwer, in schön krystallisirtem Zustande zu erhalten; sie gibt, in ätherischer Lösung mit Natrium behandelt, einen festen Kohlenwasserstoff von der wahrschein-

'Sitzungsber. der kaiserl. Akademie der Wissenschaften, Bd. LXXXIV, II. Abth. und Monatshefte für Chemie, 1881.

2 Ber. der deutschen chem. Gesellschaft, 17, S. 871 und 18, S. 1803. 
lichen Formel $\mathrm{C}_{30} \mathrm{H}_{46}$. Das Brom ist sehr fest gebunden, reagirt nicht mit Anilin oder Chinolin in der Siedehitze. Wird Metacholestol in eisessigsaurer Lösung mit Bromwasserstoff behandelt, so erhält man eine krystallisirte und scheinbar sehr reactionsfähige Verbindung von der Zusammensetzung $\mathrm{C}_{15} \mathrm{H}_{24} \mathrm{BrHBr}$. Bei der Oxydation mit Chromsäure in Eisessiglösung entsteht als einziges Reactionsproduct in vorzüglicher Ausbeute ein krystallisirender Körper von den Eigenschaiten eines Ketons, von welchem ein krystallisirtes Oxim und Hydrazon dargestellt worden ist. Weder Oxim, noch Hydrazon werden bei der Reduction in alkoholischer Lösung erheblich verändert.

Goldschmiedt. 